\title{
Using SOFIA for Stellar Physics Studies
}

\author{
Ravi Sankrit ${ }^{1}$ \\ ${ }^{1}$ SOFIA Science Center, NASA Ames Research Center, \\ Moffett Field, CA 94035, United States \\ email: rsankrit@sofia.usra.edu
}

\begin{abstract}
The Stratospheric Observatory for Infrared Astronomy (SOFIA) operates in the 0.3-240 micron wavelength region, and offers imaging, polarimetric and high-resolution spectroscopic capabilities that can be used for a wide range of observations relevant for stellar physics studies. We summarize the advantages of using SOFIA, list some of the studies related to stellar physics that have been accomplished, and provide an example high-resolution spectrum of a red supergiant.
\end{abstract}

Keywords. observatories: airborne, telescopes, infrared: stars

\section{The Observatory, Instrumentation and Capabilities}

The Stratospheric Observatory for Infrared Astronomy (SOFIA) is a joint project of NASA and the German Aerospace Center (DLR). It consists of a $2.7 \mathrm{~m}$ telescope $(2.5 \mathrm{~m}$ usable diameter) mounted in a Boeing 747-SP aircraft. SOFIA flies at altitudes up to $14 \mathrm{~km}$ (45,000 feet), above $99.8 \%$ of the water vapor in the Earth's atmosphere, and provides access to a broad wavelength range, from the optical to the far-infrared (Fig. 1). The basic characteristics of the observatory have been described by Young et al. (2012).

SOFIA can be used to establish the IR spectral energy distribution between about 4 and $200 \mu \mathrm{m}$ of sufficiently bright sources. This is a wavelength range that additionally includes a large number of atomic and molecular transitions. The angular resolution provided by SOFIA is about three times higher than Spitzer at wavelengths beyond about $15 \mu \mathrm{m}$ and comparable to that of Herschel at the longer wavelengths. SOFIA offers high-resolution spectroscopy both at mid-IR wavelengths with EXES ( $\mathrm{R}$ up to $10^{5}$ ) and at tera-hertz frequencies with GREAT ( $\mathrm{R}$ up to $10^{8}$ ). The second generation instrument $\mathrm{HAWC}+$, a far-IR camera and imaging polarimeter, will be commissioned in 2016 and provide the ability for polarization studies at wavelengths inaccessible from the ground, and which correspond to the peak emission of thermal dust in many infrared sources. SOFIA is designed to have a long life-time and is ideally suited for monitoring variable and evolving objects. As an airborne observatory, it has the flexibility to deploy to various locations to observe targets of opportunity.

The SOFIA proposal calls are open to the international astronomical community. In Observing Cycles 1-4, about $20 \%$ of the submitted proposals were in categories congruent with stellar physics. The Cycle 5 call is expected to be issued by early May 2016.

\section{SOFIA Observations relevant to Stellar Physics}

A wide range of "Stellar Physics" topics have been addressed by SOFIA observations: RV Tauri stars (Helton et al. 2015a), Born-Again-Giants (Helton et al. 2015b), Luminous Blue Variables (Lau et al. 2014), proto-planetary nebulae (Guzman-Ramirez et al. 2015), planetary nebulae (Sahai et al. 2012, Werner et al. 2014) and novae (Gehrz et al. 2015). 


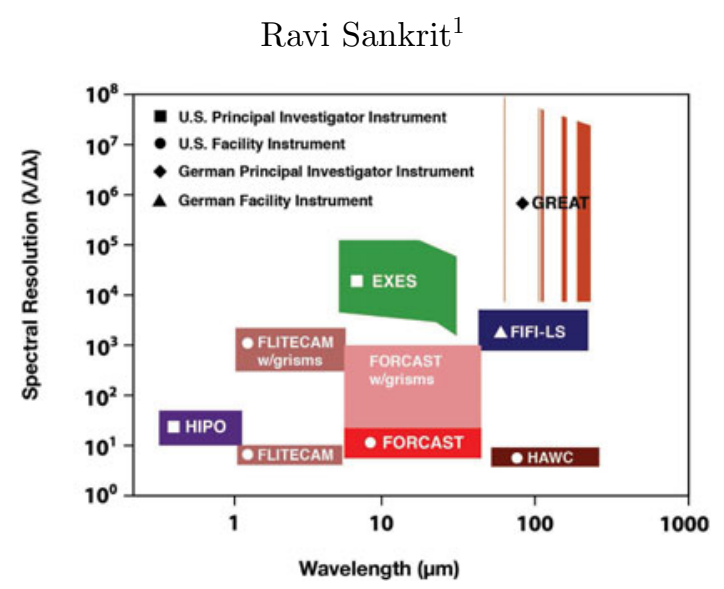

Figure 1. SOFIA instruments - wavelength coverage and spectral resolution.

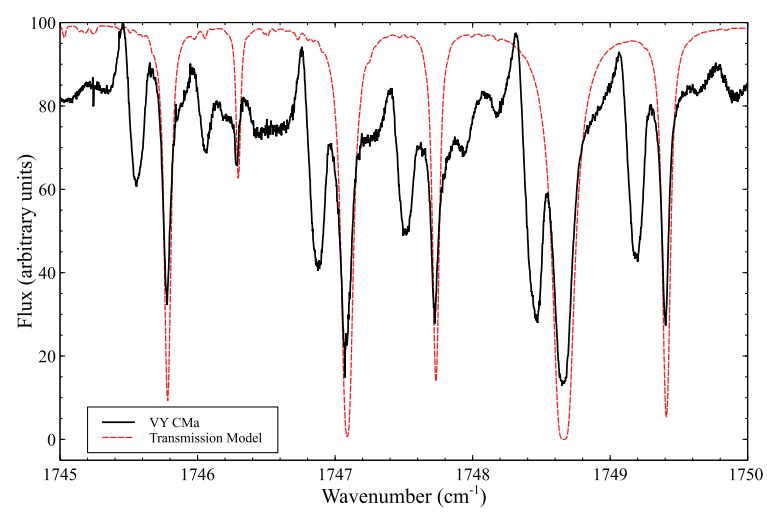

Figure 2. High-resolution (R 80,000) EXES spectrum of VY CMa, overlaid on a plot of an atmospheric transmission model. The model (red dashed line) shows the positions of the $\mathrm{H}_{2} \mathrm{O}$ absorption lines but is not an optimized fit to the observed spectrum (black solid line). Figure courtesy of C. DeWitt and the EXES team.

Here, we illustrate the power of high-resolution spectroscopy offered by SOFIA with a small portion $(5.714-5.731 \mu \mathrm{m})$ of an EXES spectrum of the red supergiant VY CMa (Fig. 2). Neufeld et al. 1999 were the first to report the detection of P Cygni profiles of $\mathrm{H}_{2} \mathrm{O}$ lines (in the range 29.5-45 $\mu \mathrm{m}$ ) from VY CMa. The P Cygni features in the EXES spectrum, red-shifted with respect to the atmospheric absorption, are due to water vapor in the supergiant envelope, and combined with data at longer wavelengths will provide strong constraints on models of the circumstellar outflow.

\section{References}

Gehrz, R. D., et al. 2015, IAU General Assembly, 22, 46143

Guzman-Ramirez, L., et al. 2015, MNRAS, 451, L1

Helton, L. A., et al. 2015a, IAU General Assembly, 22, 42535

Helton, L. A., et al. 2015b, IAU General Assembly, 22, 46157

Lau, R. M. et al. 2014, ApJ, 785, 120

Neufeld, D. A., et al. 1999, ApJL, 517, L147

Sahai, R., et al. 2012, A\&GA, 542, L20

Werner, M. W., et al. 2014, ApJ, 780, 156

Young, E. T., et al. 2012, ApJL, 749, L17 